\title{
引力波偏振
}

郜青 1 ，龚云贵 $2^{*}$, 梁迪聪

1. 西南大学物理科学与技术学院, 重庆 400715 ;

2. 华中科技大学物理学院, 武汉 430074

* 联系人, E-mail: yggong@ hust.edu.cn

2018-02-13收稿, 2018-03-09修回，2018-03-12接受，2018-03-23网络版发表

国家自然科学基金(11690021,11605061)和中央高校基本科研业务费(XDJK2017C059, SWU116053)资助

摘要 引力波的直接观测不但开启了一扇探测引力本质的全新窗口, 而且使我们进入了多信使引力波天 文学时代. 引力波不影响单个检验粒子的测地线运动, 其偏振态由两个相邻检验粒子的相对运动, 即测地线 偏离运动所决定. 测地线偏离方程中只出现黎曼张量的电分量, 它有 6 个独立分量, 所以在最一般的度规引 力理论中,引力波最多可以有6种偏振模式. 这6种偏振模式分别为: “+”偏振态、“犺”偏振态、呼吸偏振态、 矢量 $-x$ 偏振态、矢量 $-y$ 偏振态及纵振态. 爱因斯坦广义相对论中的引力波只有“+”与“ “ $\times$ ”两个张量偏振态, Brans-Dicke理论中的引力波除了“+”与“ “ $”$ 偏振态外, 还有一个呼吸偏振态. 在Horndeski及 $f(R)$ 理论中, 引 力波除了“十”与“决”两个张量偏振态外, 还有一个由呼吸模与纵振模混合而成的标量偏振态. 在爱因斯坦-以 太理论中, 非零矢量场的出现破坏了局域洛伦兹对称性, 该理论中的引力波以非光速传播, 且具有 5 个独立 偏振态. 张量-矢量-标量理论中的引力波传播速度与光速不同, 且该理论中的引力波具有 6 个独立偏振态. 因为不同引力理论中的引力波偏振态不同, 所以引力波偏振态的测量可用于探究引力本质.

关键词引引波偏振态, 标量-张量理论, 爱因斯坦-以太理论, 张量-矢量-标量理论

2016年2月11日, 美国激光干涉仪引力波天文 台(laser interferometer gravitational wave observatory, LIGO) 宣布他们在2015年9月14日观测到了距离地 球410 Mpc的两个黑洞并合时所释放的引力波信 号GW150914 ${ }^{[1]}$, 这一划时代的观测结果第一次直 接证实了引力波的存在. 它不但极大地推动了 人们对引力波的兴趣及研究热情, 而且为我们 研究引力本质开启了一扇全新的窗口。莱纳·魏 斯(Rainer Weiss)，巴里·巴里什(Barry C. Barish)和 基普·索恩(Kip S. Thorne)这三位科学家因此获得 了2017 年诺贝尔物理学奖. LIGO课题组及意大 利的Virgo课题组后来又陆续观测到了 GW151226, GW170104, GW170608, GW170814 及GW170817这 些引力波事件 ${ }^{[2 \sim 6]}$, 其中 GW170814及GW170817这
两个引力波事件还被Virgo课题组观测到了. 值得一 提的是GW170817, 它是观测到的第一个由双中子星 并合所产生的引力波, 该引力波的电磁对应体也被 世界上各种天文望远镜捕捉到了. 引力波及其电磁 对应体的直接观测真正开启了多信使引力波天文 时代.

爱因斯坦于1915年提出了广义相对论的场方程, 并且于1916年利用平直时空背景下的线性近似推 导出了引力波所满足的波动方程及引力辐射的四 极矩公式, 从而预言了引力波的存在且引力波的传 播速度为光速. 1918年爱因斯坦更正了1916年推导 的公式中的错误, 并且指出引力波只有两个独立自 由度, 即两个偏振方向. 爱因斯坦的四极矩公式表 明,一个质量分布不对称的体系在做加速运动时会

引用格式: 郜青, 龚云贵, 梁迪聪. 引力波偏振. 科学通报, 2018, 63: 801-815 Gao Q, Gong Y G, Liang D C. The polarizations of gravitational waves (in Chinese). Chin Sci Bull, 2018, 63: 801-815, doi: 10.1360/N972018-00167 
使时空产生变形, 而且这种变形会以波纹的形式向 外传播, 这就是引力波. 引力波在本质上不同于电 磁波, 由加速运动物体产生的引力波是时空纤维本 身的波. 由于引力波会扭曲时空, 会改变两个自由 宏观物体之间的距离. 但是引力波所产生的可观测 效应非常微弱, 人们一度认为不可能探测到引力波. 直到20世纪50年代, 一些相对论理论物理学家, 特别 是Bondi严格证明了引力辐射实际上是可观测的, 引 力波的探测才被重视起来. 因为引力波携带能量, 所 以一个辐射引力波的系统会损失能量, 双星系统便 是这样的系统. 双星系统中的星体相互绕转会向外 辐射引力波, 从而损耗能量, 导致双星的轨道衰减及 其周期变短. Hulse 和Taylor ${ }^{[7]}$ 通过长达30年的对脉 冲星B1913+16的观测, 发现该双星系统的轨道衰减 与广义相对论的预言惊人的一致. 该研究成果间接 地证明了引力波的存在. 1993年的诺贝尔物理学奖 颁发给了 Hulse和Taylor两人以表彰他们在此方面的 贡献.

20世纪60年代一些引力波天线被建造起来 用来探测引力波, 1969年韦伯(Joseph Weber)宣称 他利用韦伯棒探测器探测到了引力波, 但是其 结果并没有被其他研究组重复出来. Gerstenstein与Pustovoit ${ }^{[8]}$ 在 1962 年提出利用激光干涉技术 来测量引力波引起的微小偏离效应, 莱纳.魏斯 在1972年详细计算了激光干涉仪探测引力波的各 种噪声. 2002年, LIGO建造完成并开始了对引力波 的探测. 2004年, LIGO开始升级到新的先进LIGO, 并 于2015年开始运行. 先进LIGO由分别位于Livingston 及Hanford两个相距 $3002 \mathrm{~km}$ 的相同激光干涉仪构 成, 每个探测器由两个相互垂直的长度为 $4 \mathrm{~km}$ 的 干涉臂组成. 先进LIGO开始运行便探测到了引 力波信号GW150914 ${ }^{[1]}$, 从而证实了爱因斯坦广义 相对论的预言. GW170817及(1.74 \pm 0.05$) \mathrm{s}$ 后观测 到的电磁对应体伽马暴GRB170817 $\mathrm{A}^{[9,10]}$ 不仅提 供了更多的物理内涵, 而且意味着多信使引力 波天文学时代的来临. 利用这个 $(1.74 \pm 0.05) \mathrm{s}$ 的 时间差, 可以对引力波的速度给出很强的限制: $-3 \times 10^{-15} \leqslant\left(v_{G W}-v_{E M}\right) / v_{E M} \leqslant+7 \times 10^{-16[11]}$, 探 测到的引力波的速度非常接近于光速. 引力波的精 确探测, 不仅可以了解更多关于波源的信息, 同时也 提供了一个前所未有的机会来检验各种引力理论.
引力波的偏振模式是引力波理论的重要研究内 容, 同时也是引力波探测的重要方向. 由于不同的引 力理论中的引力波偏振态不尽相同, 因此对引力波 偏振态的测量将有助于甄别各种引力理论, 特别是 进一步证实爱因斯坦的广义相对论. 本文将对平直 时空背景下的引力波偏振态理论与探测进行介绍. 本文结构安排如下: 第一部分将简要介绍引力波, 并 用广义相对论中的引力波作为例子来进行说明; 第 二部分将通过测地线偏离方程来定义引力波的偏振 模式; 第三部分将探究其他的修改引力理论中的引 力波偏振态; 第四部分将简要介绍引力波偏振探测 的相关方法、理论以及已知的探测结果. 最后对本 文进行总结.

\section{1 引力波简介}

引力场和电磁场之间有很多的相似性, 通过类 比, 爱因斯坦场方程会像麦克斯韦方程一样有辐射 解. 而由于爱因斯坦场方程的高度非线性, 即引力波 本身就是一种对该引力场有贡献的能动量张量的源, 使得方程的求解十分困难. 除了寻求严格的场方程 特解, 另一种探索引力波的途径是研究场方程的远 场辐射解, 即认为引力波在某个时空背景下传播, 它 自身携带的能量和动量可以忽略不计, 从而不影响 其自身的传播. 本文采用后一种研究方法, 主要研究 在平直时空背景之下, 弱引力波的传播及其偏振态. 采用弱场线性近似, 将度规 $g_{\mu \nu}$ 分解成为闵氏度规背 景 $\eta_{\mu \nu}$ 和扰动 $h_{\mu \nu}$ 两部分:

$$
g_{\mu \nu}=\eta_{\mu \nu}+h_{\mu \nu}
$$

为了计算方便, 将在全文(第四部分除外)中都假定引 力波沿 $z$ 方向传播. 类光波矢为 $k^{\mu}$, 满足 $\eta_{\mu \nu} k^{\mu} k^{\nu}=0$.

首先考虑广义相对论中的引力波. 广义相对论 中, 引力作用量的形式为

$$
S=\frac{1}{16 \pi G} \int \mathrm{d}^{4} x \sqrt{-g} R,
$$

对度规做变分, 可得真空中的爱因斯坦场方程

$$
R_{\mu \nu}-\frac{1}{2} g_{\mu v} R=0 .
$$

在线性近似下, 黎曼张量可表达为

$$
R_{\mu \nu \alpha \beta} \approx \frac{1}{2}\left(h_{\nu \alpha, \mu \beta}+h_{\mu \beta, \nu \alpha}-h_{\mu \alpha, \nu \beta}-h_{\nu \beta, \mu \alpha}\right),
$$


由此可得到 $R_{\mu \nu}, R$ 及爱因斯坦张量 $G_{\mu \nu}$ 的一阶近似表 达式. 为了计算方便, 引入反迹变量

$$
\bar{h}_{\mu \nu}=h_{\mu \nu}-\frac{1}{2} \eta_{\mu \nu} h,
$$

则线性近似下 $G_{\mu \nu}$ 可表达为

$$
G_{\mu \nu}=\frac{1}{2}\left(\partial_{\mu} \partial_{\alpha} \bar{h}_{v}^{\alpha}+\partial_{v} \partial_{\alpha} \bar{h}_{\mu}^{\alpha}-\square \bar{h}_{\mu \nu}-\eta_{\mu \nu} \partial_{\alpha} \partial_{\beta} \bar{h}^{\alpha \beta}\right) .
$$

由于广义坐标变换的自由度, 可以选取协和坐标, 即 横向规范 $\partial_{\mu} \bar{h}^{\mu \nu}=0$. 代入到方程(6), 则线性化的真空 场方程为

$$
\square \bar{h}_{\mu \nu}=0,
$$

其解为平面波

$$
\bar{h}_{\mu \nu}=\epsilon_{\mu \nu} \exp \left(i k_{\mu} x^{\mu}\right)+c . c .,
$$

其中 $\epsilon_{\mu v}=\epsilon_{v \mu}$ 代表偏振态, 满足横波条件 $k^{\mu} \epsilon_{\mu v}=0$. 进 一步取无迹条件 $h=\eta^{\mu v} h_{\mu v}=0$, 则有 $\bar{h}_{\mu v}=h_{\mu v}$. 由此 可得, 时空的扰动 $h_{\mu \nu}$ 满足波动方程, 它是动力学的, 是可传播的, 其传播速度为光速, 这就是引力波. 在 横向无迹规范条件下, 非零分量为 $h_{x x}^{T T}=-h_{y y}^{T T}=h_{+}$, $h_{x y}^{T T}=h_{y x}^{T T}=h_{\times}$.

\section{2 引力波的偏振态}

对于电磁场, 通常用电场的方向描述电磁场的 偏振态. 但描述引力波的量是张量, 它没有直观的方 向定义, 其偏振态也就不能简单地用方向来描述了. 注意到电场的方向就是检验正电荷的运动方向, 可 以类似地考虑用检验粒子在引力场中的运动来定义 引力波的偏振态. 在广义相对论中, 自由下落的检验 粒子走测地线方程, 其对应的坐标加速度为

$$
\begin{aligned}
\frac{\mathrm{d}^{2} x^{i}}{\mathrm{~d} t^{2}}= & -\left(\Gamma_{t t}^{i}+2 \Gamma_{t j}^{i} v^{j}+\Gamma_{j k}^{i} v^{j} v^{k}\right) \\
& +v^{i}\left(\Gamma_{t t}^{t}+2 \Gamma_{t j}^{t} v^{j}+\Gamma_{j k}^{t} v^{j} v^{k}\right),
\end{aligned}
$$

在低速近似下, 坐标速度 $v^{i}=\mathrm{d} x^{i} / \mathrm{d} t \ll 1$, 再加上横 向无迹规范条件 $h_{\mu t}^{T T}$, 上述方程可近似为 ${ }^{[12]}$

$$
\frac{\mathrm{d}^{2} x^{i}}{\mathrm{~d} t^{2}} \approx-\Gamma_{t t}^{i}=-\frac{1}{2}\left(2 \partial_{t} h_{i t}^{T T}-\partial_{i} h_{t t}^{T T}\right)=0 .
$$

从方程形式上看, 引力波不会对检验粒子的测地 线运动造成影响, 所以单个检验粒子的自由落体
运动不能用来描述引力波偏振态. 但是如果考 虑两个相邻检验粒子的测地线运动, 则发现在引 力波的作用下, 它们之间将产生相对加速运动. 假设这两个相邻的自由下落粒子, 分别沿着轨 道 $x^{\mu}(\tau)$ 和 $x^{\mu}(\tau)+\delta x^{\mu}(\tau)$ 运动, 其测地线方程分别为

$$
\begin{aligned}
& \frac{\mathrm{d}^{2} x^{\mu}}{\mathrm{d} \tau^{2}}+\Gamma_{\nu \lambda}^{\mu}(x) \frac{\mathrm{d} x^{\nu}}{\mathrm{d} \tau} \frac{\mathrm{d} x^{\lambda}}{\mathrm{d} \tau}=0, \\
& \frac{\mathrm{d}^{2}\left(x^{\mu}+\delta x^{\mu}\right)}{\mathrm{d} \tau^{2}}+\Gamma_{\nu \lambda}^{\mu}(x+\delta x) \\
& \frac{\mathrm{d}\left(x^{\nu}+\delta x^{\nu}\right)}{\mathrm{d} \tau} \frac{\mathrm{d}\left(x^{\lambda}+\delta x^{\lambda}\right)}{\mathrm{d} \tau}=0,
\end{aligned}
$$

两式相减, 保留到 $\delta x^{\mu}$ 的一次项, 整理可得

$$
\frac{D^{2}}{D \tau^{2}} \delta x^{\lambda}=R^{\lambda}{ }_{\nu \mu \rho} \delta x^{\mu} \frac{\mathrm{d} x^{\nu}}{\mathrm{d} \tau} \frac{\mathrm{d} x^{\rho}}{\mathrm{d} \tau} .
$$

在局域惯性观测者坐标系(沿着第一个检验粒子的 测地线运动的坐标系)中, 两个做自由落体运动的相 邻检验粒子之间的距离满足(在后文中用 $x^{i}$ 替代 $\delta x^{i}$ )

$$
\frac{\mathrm{d}^{2} x^{i}}{\mathrm{~d} t^{2}}=R_{i 0 j 0} x^{j} .
$$

这就是局域惯性观测者坐标系中的测地线偏离方程, 表示两个相邻检验粒子将在引力波的作用下发生相 对加速运动. 注意在线性近似下 $R_{i 0 j 0}$ 是规范不变量, 因此可以利用测地线偏移方程来定义引力波偏振态. 通常把检验粒子放在一个球面上, 用这个球面上的 检验粒子在引力波作用下的运动来定义引力波偏振 态. 以下考虑广义相对论中的情况.

在横向无迹规范下, 广义相对论中的平面引力 波 $h_{\mu v}$ 具有两个独立自由度,

$$
h_{\mu \nu}=\left(\begin{array}{cccc}
0 & 0 & 0 & 0 \\
0 & \epsilon_{11} & \epsilon_{12} & 0 \\
0 & \epsilon_{12} & -\epsilon_{11} & 0 \\
0 & 0 & 0 & 0
\end{array}\right) \cos [\Omega(t-z)] .
$$

把方程(15)代入方程(4)及(14)可得, 在横向无迹规范 下，

$$
\frac{\mathrm{d}^{2} x^{i}}{\mathrm{~d} t^{2}}=-\frac{1}{2} \ddot{h}_{i j}^{T T} x^{j} .
$$

$\epsilon_{11}$ 模式引起的测地线偏离为

$$
\begin{aligned}
\ddot{x} & =\frac{\Omega^{2}}{2} \epsilon_{11} \cos [\Omega(t-z)] x, \\
\ddot{y} & =-\frac{\Omega^{2}}{2} \epsilon_{11} \cos [\Omega(t-z)] y,
\end{aligned}
$$


这通常称之为“+”偏振模式, 其振动模式见图1(a). $\epsilon_{12}$ 模式引起的测地线偏离为

$$
\begin{aligned}
& \ddot{x}=\frac{\Omega^{2}}{2} \epsilon_{12} \cos [\Omega(t-z)] y, \\
& \ddot{y}=\frac{\Omega^{2}}{2} \epsilon_{12} \cos [\Omega(t-z)] x,
\end{aligned}
$$

这通常称之为“ $x$ ”偏振模式, 其振动模式见图1(b). “+”偏振与“X”偏振模式都是横波模式.

而在更一般的度规引力理论中, $h_{\mu \nu}$ 可能有多于 两个的独立波动自由度, 因而 $R_{i 0 j 0}$ 的独立自由度可能 多于两个, 反映到测地线偏离方程上, 则说明可能有 多个偏振模式. 由于对称性, $R_{i 0 j 0}$ 最多有 6 个独立变 量. 根据方程(14), 可以定义6种基本偏振模式,

(1) “+”偏振态: $\hat{P}_{+}=-R_{x 0 x 0}+R_{y 0 y 0}=\frac{1}{2}\left(h_{x x, 00}-h_{y y, 00}\right)$.

(2) “×”偏振态: $\hat{P}_{\times}=2 R_{x 0 y 0}=-h_{x y, 00}$.

(3) 呼吸模式: $\hat{P}_{b}=R_{x 0 x 0}+R_{y 0 y 0}=-\frac{1}{2}\left(h_{x x, 00}+h_{y y, 00}\right)$.

(4) 矢量 $-x$ 模式: $\hat{P}_{x}=R_{x 0 z 0}=\frac{1}{2}\left(h_{x 0,0 z}-h_{x z, 00}\right)$.

(5) 矢量 $-y$ 模式: $\hat{P}_{y}=R_{y 0 z 0}=\frac{1}{2}\left(h_{y 0,0 z}-h_{y z, 00}\right)$.

(6) 纵模态: $\hat{P}_{l}=R_{z 0 z 0}=-\frac{1}{2}\left(h_{00, z z}+h_{z z, 00}-2 h_{0 z, 0 z}\right)$.

为了更直观地理解引力波的上述偏振态, 考虑 球面上的自由下落的检验粒子在沿 $z$ 方向传播的引 力波的作用下的相对运动, 如图1 3所示. 对于爱因 斯坦广义相对论中的引力波, 只存在图1中的“+”偏 振态 $\hat{P}_{+}=h_{+, 00}$ 与“×”偏振态 $\hat{P}_{\times}=-h_{\times, 00}$.

以上便是一般的度规引力理论中所包含的 6 种 基本偏振模式, 但在具体的引力理论中, 其偏振模式 可能包含上述6种模式的某几种, 或包含由它们组合 形成的混合模式. 利用Newman-Penrose变量 ${ }^{[13]}$, 使用 局域洛伦兹对称性对类光引力波(以光速传播的引 力波)所具有的上述 6 种偏振模式进行分类 ${ }^{[14]}$. 按照 这个分类, 对于类光引力波, 上述 6 种偏振模式为 6 个 相互独立的偏振态. 对于弱的类光平面引力波, 不 失一般性, 假设它沿 $z$ 方向传播, 则与黎曼张量6个 电分量 $R_{i 0 j 0}$ 相联系的非零独立Newman-Penrose变量 为: $\Psi_{4}=-R_{x 0 x 0}+R_{y 0 y 0}+2 i R_{x 0 y 0}, \Phi_{22}=-R_{x 0 x 0}-R_{y 0 y 0}$, $\Psi_{3}=\left(-R_{x 0 z 0}+i R_{y 0 z 0}\right) / 2$, 以及 $\Psi_{2}=-R_{z 0 z 0} / 6$. 显然 $\Psi_{4}$ 代 表 $\hat{P}_{+}$及 $\hat{P}_{\times}, \Phi_{22}$ 代表 $\hat{P}_{b}, \Psi_{3}$ 代表 $\hat{P}_{x}$ 及 $\hat{P}_{y}, \Psi_{2}$ 代表 $\hat{P}_{l}$. 在 保持沿 $z$ 轴的波矢不变的局域洛伦兹变换下, 这 些Newman-Penrose变量的变换关系为

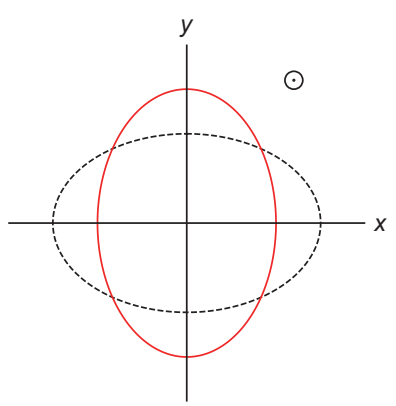

(a)

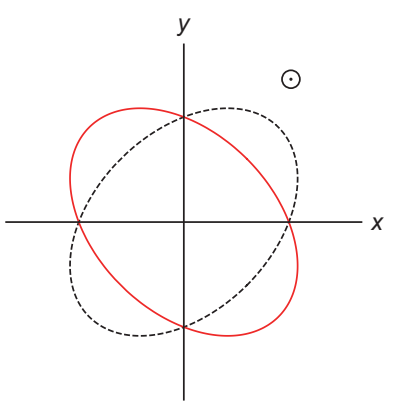

(b)
图 1 (网络版彩色)球面上的检验粒子在“+”模式与“ $\times$ ”模式引力波 作用下的位移示意图. (a) $-R_{x 0 x 0}+R_{y 0 y 0}$, 是“+”模式, 只会引起横向的 偏离; (b) $2 R_{x 0 y 0}$, 是“ “” 模式, 只会引起横向的偏离. 图右上角标示引 力波传播方向

Figure 1 (Color online) The displacement of test particles on a sphere caused by gravitational waves with the "+" and " $x$ " polarizations. (a) $-R_{x 0 x 0}+R_{y 0 y 0}$ or the "+" mode which causes the lateral deviation only; (b) $2 R_{x 0 y 0}$ or the " $\times$ " mode which causes the lateral deviation only. The symbol at the upper right in each figure shows the propagating direction of gravitational waves

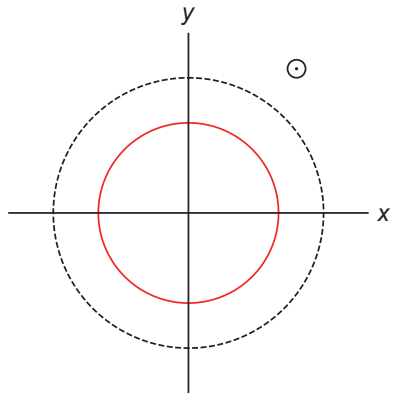

(a)

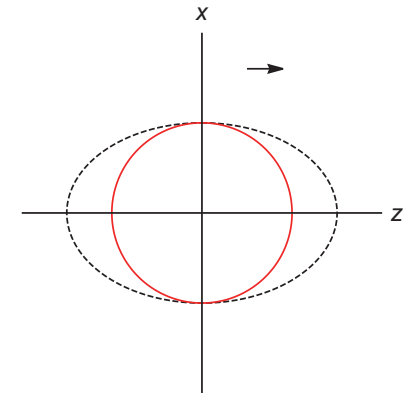

(b)
图 2 (网络版彩色)球面上的检验粒子在呼吸模式与纵振模式引力 波作用下的位移示意图. (a) $R_{x 0 x 0}+R_{y 0 y 0}$, 是呼吸模式, 可简称为“b”模 式, 只会引起横向的偏离; (b) $R_{z 0 z 0}$, 是纵振模式, 可简称为“l”模式, 只 会引起纵向的偏离. 图右上角标示引力波传播方向

Figure 2 (Color online) The displacement of test particles on a sphere caused by gravitational waves with the breathing and longitudinal polarizations. (a) $R_{x 0 x 0}+R_{y 0 y 0}$ or the breathing mode which causes the lateral deviation only; (b) $R_{z 0 z 0}$ or the longitudinal mode which causes the longitudinal deviation only. The symbol at the upper right in each figure shows the propagating direction of gravitational waves

$$
\begin{aligned}
\Psi_{2}^{\prime} & =\Psi_{2}, \\
\Psi_{3}^{\prime} & =\mathrm{e}^{-\mathrm{i} \varphi}\left(\Psi_{3}+3 \bar{\alpha} \Psi_{2}\right), \\
\Psi_{4}^{\prime} & =\mathrm{e}^{-2 i \varphi}\left(\Psi_{4}+4 \bar{\alpha} \Psi_{3}+6 \bar{\alpha}^{2} \Psi_{2}\right), \\
\Phi_{22}^{\prime} & =\Phi_{22}+2 \alpha \Psi_{3}+2 \bar{\alpha} \bar{\Psi}_{3}+6 \alpha \bar{\alpha} \Psi_{2},
\end{aligned}
$$

其中 $\varphi$ 代表绕 $z$ 轴的转动角, 复矢量 $\alpha$ 代表二维空间的 平移矢量. 如果只考虑绕 $z$ 轴的转动变换, 取 $\alpha=0$, 则 


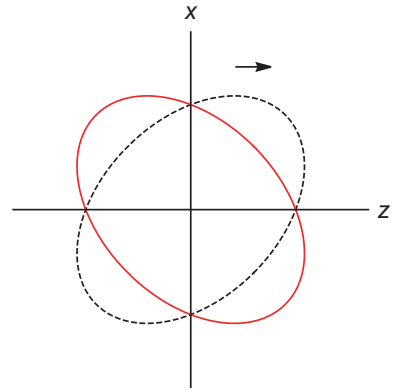

(a)

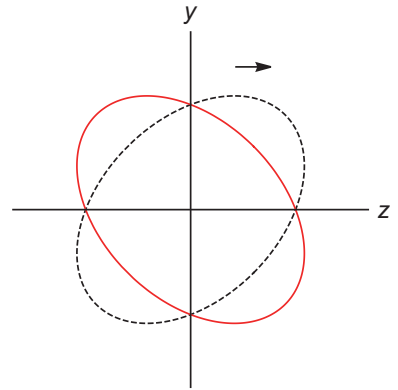

(b)
图 3 (网络版彩色) 球面上的检验粒子在矢量- $x$ 与矢量 $-y$ 模式引力波 作用下的位移示意图. (a) $R_{x 0 z 0}$, 是矢量- $x$ 模式, 同时引起横向和纵向 的偏离; (b) $R_{y 0 z 0}$, 是矢量- $y$ 模式, 同时引起横向和纵向的偏离. 图右上 角标示引力波传播方向

Figure 3 (Color online) The displacement of test particles on a sphere caused by gravitational waves with the vector- $x$ and vector- $y$ polarizations. (a) $R_{x 0 z 0}$ or the vector- $x$ mode which causes both the lateral and longitudinal deviations; (b) $R_{y 0 z}$ or the vector- $y$ mode which causes both the lateral and longitudinal deviations. The symbol at the upper right in each figure shows the propagating direction of gravitational waves

由上述变换关系(21)可知, “+”偏振模式与“×”偏 振模式代表自旋为 2 的张量引力波, 矢量- $x$ 与矢量$y$ 模式代表自旋为 1 的矢量引力波, 而呼吸模式及 纵模模式代表标量引力波. 另外, 由上述变换关 系(21)可知, 即使在某个局域惯性系中只有纵振模, 即 $\Psi_{4}=\Phi_{22}=\Psi_{3}=0$ 且 $\Psi_{2} \neq 0$, 则在变换后的局域惯 性系中, 上述变量都为非零, 所以纵振模式的出现意 味着某些局域惯性观测者应该观测到所有 6 种偏振 模式. 但该分类方法并不适用于以非光速传播的引 力波, 如 $f(R)$ 理论中的引力波 ${ }^{[15]}$ 、Horndeski理论中 的引力波 ${ }^{[16]}$ 等. 以下具体讨论修改引力中的引力波 的偏振模式.

\section{3 修改引力理论中的引力波偏振}

\subsection{Brans-Dicke理论}

1961年, Brans和Dicke ${ }^{[17]}$ 提出了Brans-Dicke理 论, 它是修改的经典引力理论之一, 也是最简单的 标量张量理论. Brans-Dicke理论从马赫原理出发, 引 入一个标量场 $\varphi$, 与度规一起传递引力相互作用. 同 时标量场的倒数等效于引力耦合强度 $G$, 其本身也可 作为暗能量的候选者. Nordtvedt ${ }^{[18]}$ 考虑了更一般的 标量张量理论中的后牛顿度规以及相关观测限制, Wagoner ${ }^{[19]}$ 计算了更一般的标量张量理论中的引力 波. 关于标量-张量引力理论的更多介绍, 参见参考
文献[20,21]. Brans-Dicke理论中的作用量为

$$
S=\frac{1}{16 \pi G} \int \mathrm{d}^{4} x \sqrt{-g}\left[\varphi R-\chi\left(\partial_{\mu} \varphi \partial^{\mu} \varphi / \varphi\right)\right],
$$

做变分, 可得真空中的场方程:

$$
\begin{aligned}
R_{\mu \nu}-\frac{1}{2} g_{\mu \nu} R= & \chi \varphi^{-2}\left(\varphi_{, \mu} \varphi_{, \nu}-\frac{1}{2} g_{\mu \nu} \varphi_{, \alpha} \varphi^{, \alpha}\right) \\
& +\varphi^{-1}\left(\varphi_{; \mu \nu}-g_{\mu \nu} g^{\alpha \delta} \varphi_{; \alpha \delta}\right)
\end{aligned}
$$

以及标量场的运动方程

$$
\square \varphi=0 .
$$

在弱场近似下, 可将标量场进行分解

$$
\varphi=\varphi_{0}+\delta \varphi
$$

则标量扰动 $\delta \varphi$ 也满足波动方程(24). 注意平直背景解 要求 $\varphi_{0}$ 为常数.

同时, 引入新变量

$$
\bar{h}_{\mu \nu}=h_{\mu \nu}-\frac{1}{2} \eta_{\mu \nu} h-\eta_{\mu \nu} \delta \varphi,
$$

并取横向无迹规范 $\partial_{\mu} \bar{h}^{\mu \nu}=0, \bar{h}=0$, 则场方程(23)可 化为

$$
\square \bar{h}_{\mu v}=0 .
$$

场方程(24) 和(27) 的平面波解为

$$
\begin{aligned}
& \bar{h}_{\mu \nu}=\epsilon_{\mu \nu} \exp \left(\mathrm{i} k_{\mu} x^{\mu}\right)+c . c ., \\
& \delta \varphi=\Phi_{1} \exp \left(\mathrm{i} k_{\mu} x^{\mu}\right)+\text { c.c.. }
\end{aligned}
$$

因此, 可以把扰动写成以下形式:

$$
h_{\mu \nu}=A^{+}(t-z) \epsilon_{\mu \nu}^{(+)}+A^{\times}(t-z) \epsilon_{\mu \nu}^{(\times)}-\eta_{\mu \nu} \delta \varphi(t-z),
$$

其中前两项正是对应于广义相对论中的“+”模式 和“决模式, 这里不再讨论. 由标量引力波 $\delta \varphi$ 引起的 测地线偏离为

$$
\begin{aligned}
\ddot{x} & =-\frac{1}{2} \delta \ddot{\varphi} x, \\
\ddot{y} & =-\frac{1}{2} \delta \ddot{\varphi} y,
\end{aligned}
$$

此即为呼吸偏振模式. 由此可见, 除了广义相对论 中两个张量偏振模式, 由于标量场的引入, BransDicke理论中的引力波会多一个呼吸模式 $\hat{P}_{b}=\delta \ddot{\varphi}$. 


\section{$3.2 f(R)$ 理论}

$f(R)$ 理论是对广义相对论的推广, 它可以用来 解释宇宙暴涨及暗能量等现象, 相关的综述性文章 见文献[22,23]. $f(R)$ 理论的作用量 ${ }^{[24]}$

$$
S=\frac{1}{16 \pi G} \int \mathrm{d}^{4} x \sqrt{-g} f(R),
$$

可以等效为标量张量引力作用量 ${ }^{[25,26]}$

$$
\begin{aligned}
S & =\frac{1}{16 \pi G} \int \mathrm{d}^{4} x \sqrt{-g}\left[f(\varphi)+(R-\varphi) f^{\prime}(\varphi)\right] \\
& =\frac{1}{16 \pi G} \int \mathrm{d}^{4} x\left[f^{\prime}(\varphi) R+f(\varphi)-\varphi f^{\prime}(\varphi)\right],
\end{aligned}
$$

其中 $f^{\prime}(\varphi)=\mathrm{d} f(\varphi) / \mathrm{d} \varphi$ 以及 $f^{\prime \prime}(\varphi) \neq 0$. 对度规以及标 量场 $\varphi$ 做变分, 可以得到场方程

$$
\begin{aligned}
G_{\mu \nu}= & \frac{1}{f^{\prime}(\varphi)}\left[\nabla_{\mu} \nabla_{v} f^{\prime}(\varphi)-g_{\mu \nu} \square f^{\prime}(\varphi)\right. \\
& \left.+\frac{1}{2} g_{\mu \nu}\left(f(\varphi)-\varphi f^{\prime}(\varphi)\right)\right], \\
\square f^{\prime}= & \frac{2}{3} f(\varphi)-\frac{1}{3} \varphi f^{\prime}(\varphi) .
\end{aligned}
$$

为了方便讨论, 取 $f(R)$ 的形式为 $f(R)=R+\alpha R^{2}$, 则场 方程可化为

$$
\begin{gathered}
G_{\mu \nu}=\frac{2 \alpha}{1+2 \alpha \varphi}\left(\nabla_{\mu} \nabla_{\nu} \varphi-g_{\mu \nu} \square \varphi-\frac{1}{4} g_{\mu \nu} \varphi^{2}\right), \\
\left(\square-m^{2}\right) \varphi=0,
\end{gathered}
$$

其中 $m^{2}=1 /(6 \alpha)$ 且 $\alpha>0$. 上述场方程的平直背景解 为 $\varphi_{0}=0$. 考虑线性扰动 $\varphi=\varphi_{0}+\delta \varphi$, 并引入变量

$$
\bar{h}_{\mu \nu}=h_{\mu v}-\frac{1}{2} \eta_{\mu v} h-2 \alpha \eta_{\mu v} \delta \varphi,
$$

则在选取横向无迹规范条件 $\partial^{\mu} \bar{h}_{\mu v}=0, \bar{h}=0$ 后, 场方 程成为

$$
\begin{gathered}
\square \bar{h}_{\mu v}=0, \\
\left(\square-m^{2}\right) \delta \varphi=0 .
\end{gathered}
$$

张量扰动 $\bar{h}_{\mu \nu}$ 与前面讨论的广义相对论及BransDicke理论中一样, 对应为“+”偏振态及“ $X$ ”偏振态. 标量扰动与Brans-Dicke理论类似, 但是这里的标量 场具有质量, 其平面波解为

$$
\delta \varphi=\Phi_{1} \exp \left(\mathrm{i} q_{\mu} x^{\mu}\right)+c . c .
$$

其中波矢 $q_{\mu}$ 满足 $\eta_{\mu \nu} q^{\mu} q^{v}=-m^{2}$, 可以取为 $q^{\mu}=$ $\left(\Omega, 0,0, \sqrt{\Omega^{2}-m^{2}}\right)$, 其对应的有质量的标量场的传 播速度为 $v=\sqrt{\Omega^{2}-m^{2}} / \Omega$. 只考虑标量扰动的贡献, 则

$$
h_{\mu \nu}=-2 \alpha \eta_{\mu \nu} \delta \varphi(v t-z) .
$$

其引起的测地线偏离为

$$
\begin{gathered}
\ddot{x}=\alpha \delta \ddot{\varphi} x, \\
\ddot{y}=\alpha \delta \ddot{\varphi} y, \\
\ddot{z}=-\alpha m^{2} \delta \varphi z=-\frac{1}{6} \delta \varphi z,
\end{gathered}
$$

即 $R_{x 0 x 0}=R_{y 0 y 0}=\alpha \delta \ddot{\varphi}, R_{z 0 z 0}=-\delta \varphi / 6$. 由此可见, 与前 面Brans-Dicke理论中的无质量标量场引起的偏振模 式不同, $f(R)$ 理论, 或与其等效的标量张量理论中的 有质量标量场, 不仅会激发呼吸偏振, 还会激发纵向 偏振. 但由于这两种偏振模式都是由同一个标量场 引起的, 所以该偏振态是一个由呼吸模与纵模混合 而成的单个偏振模式, 它只对应于一个自由度 ${ }^{[15]}$.

\subsection{Horndeski理论}

Horndeski ${ }^{[27]}$ 在1974年提出了最一般的标量张 量理论, 包括了前面讨论的广义相对论, BransDicke 理论及 $f(R)$ 理论. 虽然该理论的作用量包含 度规 $g_{\mu \nu}$ 和标量场 $\Phi$ 的高阶导数, 但其运动方程中最 多只含有二阶导数, 因此它不会出现Ostrogradsky不 稳定性问题 ${ }^{28]}$. Horndeski理论的作用量为

$$
S=\int \mathrm{d}^{4} x \sqrt{-g}\left(L_{2}+L_{3}+L_{4}+L_{5}\right)
$$

其中

$$
\begin{aligned}
L_{2}= & K(\Phi, X), \\
L_{3}=- & G_{3}(\Phi, X) \square \Phi, \\
L_{4}= & G_{4}(\Phi, X) R+G_{4, X}\left[(\square \Phi)^{2}\right. \\
& \left.-\left(\nabla_{\mu} \nabla_{\nu} \Phi\right)\left(\nabla^{\mu} \nabla^{\nu} \Phi\right)\right], \\
L_{5}= & G_{5}(\Phi, X) G_{\mu \nu} \nabla^{\mu} \nabla^{v} \Phi-\frac{1}{6} G_{5, X}\left[(\square \Phi)^{3}\right. \\
& -3(\square \Phi)\left(\nabla_{\mu} \nabla_{\nu} \Phi\right)\left(\nabla^{\mu} \nabla^{v} \Phi\right) \\
& \left.+2\left(\nabla^{\mu} \nabla_{\alpha} \Phi\right)\left(\nabla^{\alpha} \nabla_{\beta} \Phi\right)\left(\nabla^{\beta} \nabla_{\mu} \Phi\right)\right],
\end{aligned}
$$

其中 $X=-\nabla_{\mu} \Phi \nabla^{\mu} \Phi / 2, K, G_{3}, G_{4}$ 和 $G_{5}$ 是 $\Phi$ 和 $X$ 的任意 函数, 对于 $j=4,5, G_{j, X}(\Phi, X)=\partial G_{j}(\Phi, X) \partial X$. 在背景 
场 $\Phi_{0}$ 中展开

$$
\Phi=\Phi_{0}+\delta \Phi
$$

取线性近似, 则场方程可简化为 ${ }^{[16]}$

$$
\begin{aligned}
& -\frac{1}{2} K(0)+G_{4}(0) G_{\mu \nu}^{(1)}-G_{4, \Phi}(0) \\
& \left(\delta \Phi_{\mu \nu}-\eta_{\mu v} \square \delta \Phi\right)=0, \\
& K_{, \Phi}(0)+\left[K_{, X}(0)-2 G_{3, \Phi}(0)\right] \square \delta \Phi \\
& +K_{, \Phi \Phi}(0) \delta \Phi+G_{4, \Phi}(0) R^{(1)}=0,
\end{aligned}
$$

其中 $G_{4}(0)=G_{4}\left(\Phi_{0}, 0\right), K(0)=K\left(\Phi_{0}, 0\right), K_{, X}(0)$ 表 示 $K_{X}=\partial K / \partial X$ 在 $\Phi=\Phi_{0}$ 与 $X=X_{0}=0$ 处的取值, 其余同理, $G_{\mu \nu}^{(1)}$ 与 $R^{(1)}$ 表示取扰动一阶量.

为了考虑平直时空背景下的引力波, 要 求 $g_{\mu \nu}=\eta_{\mu \nu}$ 和 $\Phi=\Phi_{0}$ 是场方程(53)和(54)的解, 因此 可得 $K(0)=0$ 和 $K_{, \Phi}(0)=0$. 将此条件代入方程(53) 和(54), 可得

$$
\begin{gathered}
\left(\square-m^{2}\right) \delta \Phi=0, \\
G_{\mu \nu}^{(1)}-\sigma\left(\partial_{\mu} \partial_{\nu} \delta \Phi-\eta_{\mu \nu} \square \delta \Phi\right)=0,
\end{gathered}
$$

其中标量场质量

$$
m^{2}=-\frac{K_{, \Phi \Phi}(0)}{K_{, X}(0)-2 G_{3, \Phi}(0)+3 G_{4, \Phi}^{2}(0) / G_{4}(0)},
$$

$\sigma=G_{4, \Phi}(0) / G_{4}(0)$ 且 $G_{4}(0) \neq 0$. 和前面的讨论类似, 引入新变量

$$
\bar{h}_{\mu \nu}=h_{\mu \nu}-\frac{1}{2} \eta_{\mu \nu} h-\eta_{\mu \nu} \sigma \delta \Phi
$$

代入场方程, 并取横向无迹规范 $\partial^{\mu} \bar{h}_{\mu \nu}=0, \bar{h}=0$, 可 得

$$
\begin{gathered}
\square \bar{h}_{\mu \nu}=0, \\
\left(\square-m^{2}\right) \delta \Phi=0 .
\end{gathered}
$$

与 $f(R)$ 理论类似, Horndeski理论中的有质量的标量 场, 也将产生一个由呼吸模与纵向模混合而成的 偏振模式. 假设标量场的波矢为 $q^{\mu}=\left(q_{t}, 0,0, q_{z}\right)$, 则 $q_{t}^{2}=m^{2}+q_{z}^{2}$. 标量引力波引起的测地线偏离为 ${ }^{[16]}$

$$
\begin{aligned}
& \ddot{x}=\frac{1}{2} q_{t}^{2} \sigma \delta \Phi x, \\
& \ddot{y}=\frac{1}{2} q_{t}^{2} \sigma \delta \Phi y, \\
& \ddot{z}=\frac{1}{2} m^{2} \sigma \delta \Phi z .
\end{aligned}
$$

由于纵向的偏离正比于标量引力子质量平 方 $m^{2}$ 且 $m^{2}<q_{t}^{2}$, 所以纵振模相对于呼吸模式有一 个压低效应. 当质量为零时, 引力波以光速传播, 呼 吸模式 $\hat{P}_{\mathrm{b}}$ 和纵振模式 $\hat{P}_{1}$ 为两个独立的偏振态 ${ }^{[14]}$. 但 在Horndeski理论中, 纵振模式相对呼吸模式有一个 标量引力子质量平方的因子, 所以在质量为零时只 有呼吸模式 $\hat{P}_{\mathrm{b}}$. 当标量场具有非零质量时, 其对应 的标量引力波传播速度不再是光速, 其激发的呼吸 模 $\hat{P}_{\mathrm{b}}$ 与纵模 $\hat{P}_{1}$ 不再是两个独立的偏振态, 而是一个 独立单态, 把这样的态称为混合单态 ${ }^{[15,16]}$.

\section{4 爱因斯坦-以太理论}

量子引力可能破坏洛伦兹对称性, 关于破坏洛 伦兹不变性的相关研究工作可参考Mattingly ${ }^{[29]}$ 的综 述性文章. 爱因斯坦-以太理论通过引入一个叫做以 太场的单位类时矢量场来破坏局域的洛伦兹不变 性 ${ }^{[30]}$. 这个动力学的以太场, 定义了一个特殊的局域 参考系, 但仍然具有广义协变性 ${ }^{[30 ~ 33]}$. 关于爱因斯 坦-以太理论的相关研究工作及观测限制, 可参考文 献[34 42]. 爱因斯坦-以太理论中的引力波以非光速 传播, 具有 5 个独立偏振态 ${ }^{[43]}$. 本节利用与规范无关 的方法来分析爱因斯坦-以太理论中的引力波.

爱因斯坦-以太理论的作用量为

$$
\begin{aligned}
S= & \int \mathrm{d}^{4} x \sqrt{-g}\left[R-c_{1}\left(\nabla_{\mu} u_{v}\right) \nabla^{\mu} u^{v}\right. \\
& -c_{2}\left(\nabla_{\mu} u^{\mu}\right)^{2}-c_{3}\left(\nabla_{\mu} u_{v}\right) \nabla^{v} u^{\mu} \\
& \left.+c_{4}\left(u^{\rho} \nabla_{\rho} u^{\mu}\right) u^{\sigma} \nabla_{\sigma} u_{\mu}+\lambda\left(u^{\mu} u_{\mu}+1\right)\right],
\end{aligned}
$$

真空场方程为

$$
R_{\mu \nu}-\frac{1}{2} g_{\mu \nu} R=T_{\mu \nu}^{e}
$$

其中

$$
\begin{aligned}
T_{\mu v}^{e}= & \lambda\left[u_{\mu} u_{v}-\frac{1}{2} g_{\mu v}\left(u^{\rho} u_{\rho}+1\right)\right] \\
& +c_{1}\left[\left(\nabla_{\mu} u_{\rho}\right) \nabla_{v} u^{\rho}-\left(\nabla_{\rho} u_{\mu}\right) \nabla^{\rho} u_{v}\right. \\
& \left.+\nabla_{\rho}\left(u_{(\mu} \nabla^{\rho} u_{v)}-u_{(\mu} \nabla_{v)} u^{\rho}\right)+u^{\rho} \nabla_{(\mu} u_{v)}\right] \\
& +c_{2} g_{\mu v} \nabla_{\rho}\left(u^{\rho} \nabla_{\sigma} u^{\sigma}\right)+c_{3} \nabla_{\rho}\left[u_{(\mu} \nabla_{v)} u^{\rho}\right. \\
& \left.-u_{(\mu} \nabla^{\rho} u_{v)}+u^{\rho} \nabla_{(\mu} u_{v)}\right] \\
+ & c_{4}\left[a_{\mu} a_{v}-\nabla_{\rho}\left(2 u^{\rho} u_{(\mu} a_{v)}-a^{\rho} u_{\mu} u_{v}\right)\right] \\
+ & \frac{1}{2} g_{\mu v}\left[-c_{1}\left(\nabla_{\rho} u_{\sigma}\right) \nabla^{\rho} u^{\sigma}-c_{2}\left(\nabla_{\rho} u^{\rho}\right)^{2}\right.
\end{aligned}
$$




$$
\left.-c_{3}\left(\nabla_{\rho} u_{\sigma}\right) \nabla^{\sigma} u^{\rho}+c_{4} a_{\rho} a^{\rho}\right]
$$

以及 $a^{\mu}=u^{\nu} \nabla_{v} u^{\mu}$. 矢量场 $u^{\mu}$ 的背景解为 $\bar{u}^{\mu}=$ $(1,0,0,0)$. 由于背景解不为零, 它定义了一个确定 方向, 即选择了一个特殊惯性系, 从而破坏了局域洛 伦兹不变性. 以太场在此背景中的线性展开为

$$
u^{\mu}=\bar{u}^{\mu}+v^{\mu} .
$$

先把度规扰动 $h_{\mu \nu}$ 以及以太场扰动 $v^{\mu}$ 进行标量、矢量 及张量分解 ${ }^{[12]}$,

$$
\begin{gathered}
h_{t t}=2 \Phi, \\
h_{t j}=\beta_{j}+\partial_{j} \Gamma, \\
h_{j k}=h_{j k}^{\mathrm{TT}}+\frac{1}{3} H \delta_{j k}+\partial_{(j} \epsilon_{k)}+\left(\partial_{j} \partial_{k}-\frac{1}{3} \delta_{j k} \nabla^{2}\right) \rho, \\
v^{0}=\frac{1}{2} h_{t t}=\Phi, \\
v^{j}=\mu^{j}+\partial^{j} V,
\end{gathered}
$$

其中 $h_{j k}^{\mathrm{TT}}$ 是 $h_{j k}$ 的横向无迹部分, 满足方程 $\partial^{j} h_{j k}^{T T}=$ $\delta^{j k} h_{j k}^{T T}=0, \beta_{j}, \epsilon_{j}$ 和 $\mu^{j}$ 都是横向矢量, 满足方 程 $\partial^{j} \beta_{j}=\partial^{j} \epsilon_{j}=\partial_{j} \mu^{j}=0$. 其中式(71)是由归一化 条件 $u^{\mu} u_{\mu}=-1$ 得到的.

在无穷小坐标变换 $x_{\mu} \rightarrow x_{\mu}+\xi_{\mu}$ 下, 有

$$
\begin{gathered}
h_{\mu v} \rightarrow h_{\mu v}-\partial_{\mu} \xi_{v}-\partial_{\nu} \xi_{\mu}, \\
u^{\mu} \rightarrow u^{\mu}+\bar{u}^{v} \partial_{\nu} \xi^{\mu} .
\end{gathered}
$$

如果把无穷小坐标变换作这样的分解 $\xi_{\mu}=\left(\xi_{t}, \xi_{j}\right)=$ $\left(A, B_{j}+\partial_{j} C\right)$ 且 $\partial^{j} B_{j}=0$, 则利用上述分解及坐标变换 性质, 可以得到这些分量的坐标变换关系 ${ }^{[12]}$

$$
\begin{gathered}
\Phi \rightarrow \Phi-\dot{A}, \Gamma \rightarrow \Gamma-A-\dot{C}, H \rightarrow H-2 \nabla^{2} C, \\
\rho \rightarrow \rho-2 C, V \rightarrow V+\dot{C}, \\
\beta_{j} \rightarrow \beta_{j}-\dot{B}_{j}, \epsilon_{j} \rightarrow \epsilon_{j}-2 B_{j}, \mu^{j} \rightarrow \mu^{j}+\dot{B}^{j}, \\
h_{i j}^{\mathrm{TT}} \rightarrow h_{i j}^{\mathrm{TT}} .
\end{gathered}
$$

显然 $h_{i j}^{\mathrm{TT}}$ 为规范不变量, 由此还可以引入以下规范不 变量 ${ }^{[44]}$

$$
\begin{gathered}
\Pi=-\Phi+\dot{\Gamma}-\frac{1}{2} \ddot{\rho}, \\
\Lambda=\frac{1}{3}\left(H-\nabla^{2} \rho\right),
\end{gathered}
$$

$$
\begin{gathered}
\Xi_{j}=\beta_{j}-\frac{1}{2} \dot{\epsilon}_{j}, \\
\Sigma_{j}=\beta_{j}+\mu_{j}, \\
\Psi=V+\frac{1}{2} \dot{\rho} .
\end{gathered}
$$

把这些规范不变量代入到场方程, 并进行化简, 可以 得到

$$
\begin{gathered}
\ddot{\Psi}-s_{s}^{2} \nabla^{2} \psi=0, \\
\ddot{\Sigma}_{j}-s_{v}^{2} \nabla^{2} \Sigma_{j}=0, \\
\ddot{h}_{i j}^{\mathrm{TT}}-s_{g}^{2} \nabla^{2} h_{i j}^{\mathrm{TT}}=0,
\end{gathered}
$$

其中的传播速度表达式分别为

$$
\begin{gathered}
s_{g}^{2}=\frac{1}{1-c_{13}}, \\
s_{v}^{2}=\frac{c_{1}-c_{1}^{2} / 2+c_{3}^{2} / 2}{c_{14}\left(1-c_{13}\right)}, \\
s_{s}^{2}=\frac{c_{123}\left(2-c_{14}\right)}{c_{14}\left(1-c_{13}\right)\left(2+2 c_{2}+c_{123}\right)},
\end{gathered}
$$

$c_{14}=c_{1}+c_{4}, c_{123}=c_{1}+c_{2}+c_{3}$, 其他依此类推. 在上 述方程推导过程中, 为避免奇点及零点, 选取了以下 条件,

$c_{13} \neq 1, \quad c_{14} \neq 0, \quad c_{14} \neq 2, \quad 3 c_{2} \neq-2-c_{13}$.

其他几个规范不变量并非独立变量, 它们可表达为

$$
\begin{gathered}
\Pi=\frac{c_{14}-2 c_{13}}{2-c_{14}} \dot{\Psi}, \\
\Lambda=\frac{2 c_{14}\left(c_{13}-1\right)}{2-c_{14}} \dot{\Psi}, \\
\Xi_{j}=-\frac{c_{13}}{1-c_{13}} \Sigma_{j} .
\end{gathered}
$$

所以爱因斯坦-以太理论中的引力波有两个张量自 由度 $h_{i j}^{T T}$, 两个矢量自由度 $\Sigma_{j}$ 及一个标量自由度 $\Psi$, 这些引力波的传播速度一般都不是光速. 张量模 式 $h_{i j}^{\mathrm{TT}}$ 与广义相对论中的引力波类似, 其非零部分 为 $h_{11}^{\mathrm{TT}}=-h_{22}^{\mathrm{TT}}=h_{+}$和 $h_{12}^{\mathrm{TT}}=h_{21}^{\mathrm{TT}}=h_{\times}$. 由于已经假定 了引力波沿 $+z$ 方向传播, 那么标量模式、矢量模式 以及张量模式的波矢可分别写成

$$
\begin{aligned}
& k_{s}^{\mu}=\Omega_{s}\left(1,0,0,1 / s_{s}\right), \\
& k_{v}^{\mu}=\Omega_{v}\left(1,0,0,1 / s_{v}\right), \\
& k_{g}^{\mu}=\Omega_{g}\left(1,0,0,1 / s_{g}\right) .
\end{aligned}
$$


在矢量模式中, 因为 $\partial_{j} \Sigma^{j}=0$, 所以有 $\Sigma_{3}=0$. 引入规 范 $h_{0 j}=0$ 及 $v^{j}{ }_{j}=0$, 可以得到以下 5 个独立的可传播 的自由度 ${ }^{[44]}$

$$
\begin{gathered}
h_{+}=\epsilon_{11} \cos \left[\Omega_{g}\left(t-z / s_{g}\right)\right], \\
h_{\times}=\epsilon_{12} \cos \left[\Omega_{g}\left(t-z / s_{g}\right)\right], \\
v_{j}=\mu_{j}^{0} \cos \left[\Omega_{v}\left(t-z / s_{v}\right)\right], \quad j=1,2, \\
\Phi=\varphi \cos \left[\Omega_{s}\left(t-z / s_{s}\right)\right] .
\end{gathered}
$$

张量模式 $h_{+}$及 $h_{\times}$分别对应 + 偏振态及 $\times$偏振态. 矢量 模式及标量模式对度规扰动 $h_{\mu v}$ 的贡献为

$$
\begin{aligned}
h_{00}= & 2 \Phi \equiv \epsilon_{00} \cos \left[\Omega_{s}\left(t-z / s_{s}\right)\right], \\
h_{j 3}= & \frac{c_{13}}{\left(c_{13}-1\right) s_{v}} v_{j} \equiv \epsilon_{j 3} \cos \left[\Omega_{v}\left(t-z / s_{v}\right)\right], \\
& j=1,2 \\
h_{11}^{t r}= & h_{22}^{t r}=c_{14} \Phi \equiv a \epsilon_{00} \cos \left[\Omega_{s}\left(t-z / s_{s}\right)\right], \\
h_{33}= & -\frac{2 c_{14}\left(1+c_{2}\right)}{c_{123}} \Phi \equiv b \epsilon_{00} \cos \left[\Omega_{s}\left(t-z / s_{s}\right)\right],
\end{aligned}
$$

其中 $a=c_{14} / 2, b=-c_{14}\left(1+c_{2}\right) / c_{123}$. 以下考虑矢量模 式 $\epsilon_{13}$ 及 $\epsilon_{23}$ 的偏振态. $\epsilon_{13}$ 模式引起的测地线偏离为

$$
\begin{aligned}
& \ddot{x}=\frac{\Omega_{v}^{2}}{2} \epsilon_{13} \cos \left[\Omega_{v}\left(t-z / s_{v}\right)\right] z, \\
& \ddot{z}=\frac{\Omega_{v}^{2}}{2} \epsilon_{13} \cos \left[\Omega_{v}\left(t-z / s_{v}\right)\right] x,
\end{aligned}
$$

此即为矢量 $-x$ 模式 $\hat{P}_{x} . \epsilon_{23}$ 模式引起的测地线偏离为

$$
\begin{aligned}
& \ddot{y}=\frac{\Omega_{v}^{2}}{2} \epsilon_{23} \cos \left[\Omega_{v}\left(t-z / s_{v}\right)\right] z, \\
& \ddot{z}=\frac{\Omega_{v}^{2}}{2} \epsilon_{23} \cos \left[\Omega_{v}\left(t-z / s_{v}\right)\right] y,
\end{aligned}
$$

此即为矢量 $-y$ 模式 $\hat{P}_{y}$. 最后讨论标量模式 $\epsilon_{00}$ 的偏振 态. $\epsilon_{00}$ 引起的测地线偏离为

$$
\begin{array}{r}
\ddot{x}=\frac{\Omega_{s}^{2}}{2} a \epsilon_{00} \cos \left[\Omega_{s}\left(t-z / s_{s}\right)\right] x, \\
\ddot{y}=\frac{\Omega_{s}^{2}}{2} a \epsilon_{00} \cos \left[\Omega_{s}\left(t-z / s_{s}\right)\right] y, \\
\ddot{z}=\frac{\Omega_{s}^{2}}{2}\left(b+\frac{1}{s_{s}^{2}}\right) \epsilon_{00} \cos \left[\Omega_{s}\left(t-z / s_{s}\right)\right] z .
\end{array}
$$

此即呼吸态 $\hat{P}_{\mathrm{b}}$ 与纵模态 $\hat{P}_{1}$ 的混合单态. 由此可见, 在爱因斯坦-以太理论中, 虽然标量场是无质量
的, 但标量引力波的传播速度不是光速, 与 $f(R)$ 以 及Horndeski理论中的有质量标量场类似, 它对应的 是呼吸模式和纵向模式组合而成的混合单态. 综 合上述结果, 爱因斯坦-以太理论中的引力波有以 下 5 个偏振态: + 偏振态 $\hat{P}_{+}$及 $\times$偏振态 $\hat{P}_{\times}$, 它们的传播 速度为 $s_{g}$; 矢量 $-x$ 模式 $\hat{P}_{x}$ 与矢量 $-y$ 模式 $\hat{P}_{y}$, 它们的传 播速度为 $s_{v}$; 呼吸态 $\hat{P}_{\mathrm{b}}$ 与纵模态 $\hat{P}_{1}$ 的混合单态, 它的 传播速度为 $s_{s}$.

\section{5 张量-矢量-标量理论}

张量-矢量-标量(tensor-vector-scalar, TeVeS)理论 是Bekenstein ${ }^{[45]}$ 在2004年提出来的张量-矢量-标量引 力理论, 它是修改的牛顿力学(modified newtonian dynamics, MOND ${ }^{[46 \sim 48]}$ ) 的相对论性推广. 与爱因斯坦以态理论不同, TeVeS理论是一个双度规理论. 该理 论引入一个度规场 $g_{\mu v}$, 也叫作爱因斯坦度规, 一个类 时的矢量场 $A_{\mu}$ 以及一个标量场 $\varphi$ 来传递引力相互作 用. 而物质场与另一个度规, 也就是物理度规进行耦 合. 该理论中的物理度规为

$$
\tilde{g}_{\alpha \beta}=\mathrm{e}^{-2 \varphi} g_{\alpha \beta}-2 A_{\alpha} A_{\beta} \sinh (2 \varphi) .
$$

$\mathrm{Sagi}^{[49]}$ 研究了 $\mathrm{TeVeS}$ 理论中的引力波, 并给出了引力 波的偏振态及其传播速度. 关于该理论的其他相关 研究工作, 可参考文献[50 53].

$\mathrm{TeVeS}$ 理论中的度规场、物质场、矢量场以及 标量场的作用量分别为 ${ }^{[49]}$

$$
\begin{array}{r}
S_{g}=\frac{1}{16 \pi G} \int g^{\alpha \beta} R_{\alpha \beta} \sqrt{-g} \mathrm{~d}^{4} x, \\
S_{m}=\int L\left(\tilde{g}_{\mu \nu}, f^{\alpha}, f_{; \mu}^{\alpha}, \cdots\right) \sqrt{-\tilde{g}} \mathrm{~d}^{4} x,
\end{array}
$$

$$
\begin{aligned}
S_{v}= & -\frac{1}{16 \pi G} \int \sqrt{-g} \mathrm{~d}^{4} x\left[\frac{K}{2} F_{\alpha \beta} F^{\alpha \beta}+\frac{K_{+}}{2} S_{\alpha \beta} S^{\alpha \beta}\right. \\
& \left.+K_{2}(\nabla A)^{2}+K_{4} \dot{A}_{\alpha} \dot{A}^{\alpha}-\zeta\left(A^{\alpha} A_{\alpha}+1\right)\right] \\
S_{s}= & -\frac{1}{2 k^{2} l^{2} G} \int F\left(k l^{2} j^{\alpha \beta} \varphi_{, \alpha} \varphi_{, \beta}\right) \sqrt{-g} \mathrm{~d}^{4} x
\end{aligned}
$$

其中 $F_{\alpha \beta}=A_{\alpha ; \beta}-A_{\beta ; \alpha}, S_{\alpha \beta}=A_{\alpha ; \beta}+A_{\beta ; \alpha}, \dot{A}^{\alpha}=A^{\beta} A_{; \beta}^{\alpha}$, $j^{\alpha \beta} \equiv g^{\alpha \beta}-A^{\alpha} A^{\beta}, A^{\alpha} \equiv g^{\alpha \beta} A_{\beta}, K_{i}$ 和 $K$ 是耦合常数, $k$ 是 无量纲参数, $l$ 是常数, $F(x)$ 是任意函数. 度规场与矢 量场的作用量与爱因斯坦-以太理论中相同, 所以它 
们给出的引力波与爱因斯坦-以太理论中的引力波 相同, 这里不再讨论. 下面只讨论标量场 $\varphi$ 激发的引 力波.

标量场所满足的场方程为

$$
\left[F^{\prime}(y) j^{\alpha \beta} \varphi_{, \alpha}\right]_{; \beta}=0 .
$$

其背景解为 $\varphi_{B}=\varphi_{c}=$ 常数. 把标量场对这个背景作 线性展开

$$
\varphi=\varphi_{B}+\delta \varphi,
$$

则得到标量扰动所满足的波动方程 ${ }^{[44]}$

$$
-\delta \ddot{\varphi}+s_{\varphi}^{2} \nabla^{2} \delta \varphi=0,
$$

其中传播速度 $s_{\varphi}^{2}=1 / 2$. 该标量扰动 $\delta \varphi$ 对物理度规扰 动的贡献为 $\tilde{h}_{00}=-2 \delta \varphi$ 及 $\tilde{h}_{i j}=-2 \delta_{i j} \delta \varphi$, 其对应的偏 振态为呼吸模 $\hat{P}_{\mathrm{b}}=-2 \delta \ddot{\varphi}$ 与纵模

$$
\hat{P}_{1}=\left(1+\frac{1}{s_{4}^{2}}\right) \delta \ddot{\varphi}
$$

的混合单态, 这里 $s_{4}=\mathrm{e}^{-2 \varphi_{c}} s_{\varphi}$ 代表该标量引力波在物 理度规中的传播速度. 在物理度规中, 其他引力波的 速度为

$$
\begin{gathered}
s_{1}^{2}=\mathrm{e}^{-4 \varphi_{c}} s_{g}^{2}, \\
s_{2}^{2}=\mathrm{e}^{-4 \varphi_{c}} s_{v}^{2}, \\
s_{3}^{2}=\mathrm{e}^{-4 \varphi_{c}} s_{s}^{2} .
\end{gathered}
$$

综上所述, TeVeS理论中的引力波有 6 个偏振态: + 偏振态 $\hat{P}_{+}$及 $\times$偏振态 $\hat{P}_{\times}$, 它们在物理度规中的传播 速度为 $s_{1}$; 矢量 $-x$ 模式 $\hat{P}_{x}$ 与矢量 $-y$ 模式 $\hat{P}_{y}$, 它们在物 理度规中的传播速度为 $s_{2}$; 两个标量自由度分别激 发的呼吸态 $\hat{P}_{b}$ 与纵模态 $\hat{P}_{l}$ 的混合单态, 它们在物理 度规中的传播速度分别为 $s_{3}$ 与 $s_{4}$.

\section{4 引力波偏振的探测}

在局域惯性观测者坐标系中, 观测者位于坐标 原点, 第二个检验粒子的坐标为 $L^{\mu}=\left(0, L^{i}\right)$. 由测地 线偏离方程(14)可得

$$
\frac{\mathrm{d}^{2} L^{i}(t)}{\mathrm{d} t^{2}}=R_{i 0 j 0} L^{j}(0)
$$

对于爱因斯坦广义相对论中的引力波, 在横向无迹 规范下, 方程(123)成为

$$
\frac{\mathrm{d}^{2} L^{i}(t)}{\mathrm{d} t^{2}}=-\frac{1}{2} \ddot{h}_{i j}^{T T} L^{j}(0) .
$$

用解 $L^{i}(t)=L_{0}^{i}+\delta L^{i}(t)$ 代入方程(124), 并假设 $|\delta L| \ll$ $\left|L_{0}\right|$, 则得到引力波引起的相对距离变化

$$
\delta L^{i}(t)=-\frac{1}{2} h_{i j}^{T T}(t) L^{j}(0) .
$$

目前, 对引力波的直接探测, 主要有共振探测、 激光干涉和脉冲星计时阵列(pulsar timing array, PTA)等方法. 脉冲星计时阵列方法主要探测频段 为 $10^{-10} \sim 10^{-6} \mathrm{~Hz}$ 的低频引力波 ${ }^{[54 ~ 59]}$, 地面引力波 探测器主要对频段为 $10 \sim 10^{4} \mathrm{~Hz}$ 的高频引力波敏 感, 而空间引力波天线如LISA(laser interferometer space antenna $)^{[60]}$, DECIGO(deci-hertz interferometer gravitational wave observatory) ${ }^{[61]}$, MAGIS(mid-band atomic gravitational wave interferometric sensor $)^{[62]}$, 天 琴[63] 与太极等主要探测频段为 $10^{-4} \sim 10 \mathrm{~Hz}$ 的中频 引力波. 这些观测手段相互补充, 通过多波段引力波 探测为研究引力本质及相关物理提供了全新的手段.

以下简单介绍激光干涉和脉冲星计时阵列这两 种探测引力波偏振的方法.

\section{1 激光干涉仪引力波天线}

假设探测器位于坐标原点, 而且两臂分别指 向 $x$ 和 $y$ 方向. 探测器张量是描述激光干涉仪探测器 的响应, 它将引力波信号转换为探测器信号, 其定义 为

$$
D^{i j}=\frac{1}{2}\left[\hat{x}_{i} \hat{x}_{j}-\hat{y}_{i} \hat{y}_{j}\right],
$$

其中 $\hat{x}$ 和 $\hat{y}$ 分别表示 $x$ 和 $y$ 方向上的单位矢量. 假设引 力波沿 $\hat{w}$ 方向传播, 那么定义引力波的偏振张量 $\epsilon_{i j}^{A}$

$$
\begin{aligned}
& \epsilon_{i j}^{+}=\hat{m}_{i} \hat{m}_{j}-\hat{n}_{i} \hat{n}_{j}, \\
& \epsilon_{i j}^{\times}=\hat{m}_{i} \hat{n}_{j}+\hat{n}_{i} \hat{m}_{j}, \\
& \epsilon_{i j}^{x}=\hat{m}_{i} \hat{w}_{j}+\hat{w}_{i} \hat{m}_{j}, \\
& \epsilon_{i j}^{y}=\hat{n}_{i} \hat{w}_{j}+\hat{w}_{i} \hat{n}_{j}, \\
& \epsilon_{i j}^{b}=\hat{m}_{i} \hat{m}_{j}+\hat{n}_{i} \hat{n}_{j}, \\
& \epsilon_{i j}^{l}=\hat{w}_{i} \hat{w}_{j},
\end{aligned}
$$

其中

$$
\hat{m}=(\cos \Phi \cos \Psi-\cos \Theta \sin \Phi \sin \Psi,
$$




$$
\begin{aligned}
& \cos \Psi \sin \Phi+\cos \Phi \cos \Theta \sin \Psi, \\
& \sin \Psi \sin \Theta), \\
\hat{n}= & (-\cos \Psi \cos \Theta \sin \Phi-\cos \Phi \sin \Psi, \\
& \cos \Phi \cos \Psi \cos \Theta-\sin \Phi \sin \Psi, \\
& \cos \Psi \sin \Theta), \\
\hat{w}=( & (\sin \Phi \sin \Theta,-\cos \Phi \sin \Theta, \cos \Theta),
\end{aligned}
$$

其中 $\Theta, \Phi$ 和 $\Psi$ 表示坐标系 $\{x, y, z\}$ 与坐标系 $\{m, n, w\}$ 之 间的转动欧拉角. 由此可以定义探测器的模式函 数 $F^{A}$

$$
F^{A}(\hat{w})=D^{i j} \epsilon_{i j}^{A}(\hat{w}) .
$$

对于每个偏振模式, 其对应的模式函数为

$$
\begin{aligned}
F^{+}= & \frac{1}{2}\left(1+\cos ^{2} \Theta\right) \cos 2 \Phi \cos 2 \Psi \\
& -\cos \Theta \sin 2 \Phi \sin 2 \Psi, \\
F^{\times}= & -\frac{1}{2}\left(1+\cos ^{2} \Theta\right) \cos 2 \Phi \sin 2 \Psi \\
& -\cos \Theta \sin 2 \Phi \cos 2 \Psi, \\
F^{x}= & \sin \Theta(\sin 2 \Phi \cos \Psi+\cos \Theta \cos 2 \Phi \sin \Psi), \\
F^{y}= & \sin \Theta(-\sin 2 \Phi \sin \Psi \\
& +\cos \Theta \cos 2 \Phi \cos \Psi), \\
F^{\mathrm{b}}= & \frac{1}{2} \cos 2 \Phi \sin ^{2} \Theta, \\
F^{1}= & -\frac{1}{2} \cos 2 \Phi \sin ^{2} \Theta
\end{aligned}
$$

可见, 因为呼吸模式和纵振模式的探测器模式函数 仅差一个负号, 所以如果用传统的两臂互相垂直的 激光干涉仪无法区分这两种模式. 此外, 对于如双星 并合等的短暂引力波信号, 至少需要 5 个非共向探测 器才能消除所有的简并度 ${ }^{[64,65]}$.

现有探测器可通过测量持续足够长时间的信号 来分析其偏振信息 ${ }^{[66 ~ 69]}$. 快速旋转的中子星是重要 的连续引力波波源 ${ }^{[70,71]}$, 其辐射的引力波在 $\mathrm{LIGO}$ 等 探测器的灵敏频段内. 对于这种连续引力波信号, 可以在一个很长的时间周期内对信号做积分, 从 而提高其探测可能性. 此外, 这种引力波信号接近 于正弦信号, 而且频率稳定, 可以通过关注其幅度 调制而提取偏振态的信息. 目前LIGO及Virgo合作 组通过对 200 颗已知脉冲星的连续引力波信号的搜 寻, 并没有发现任何引力波偏振信号, 并且他们给
出在 $95 \%$ 置信度上, 矢量及标量引力波的强度 $h$ 应小 于1.5 $\times 10^{-26[72]}$. LIGO及Virgo合作组对GW170814的 观测结果则更倾向于纯张量引力波, 而非纯矢量及 纯标量引力波 ${ }^{[5]}$.

\section{2 脉冲星计时阵列探测}

除了利用激光干涉仪来探测引力波外, 还 可以利用脉冲星计时阵列方法来探测引力波. 脉冲星是宇宙中最精准的时钟之一, 其高度周 期性为探究引力波对脉冲星信号的影响提供 了机会. PTA方法可以区分引力波的呼吸模式 和纵向模式. 1975年Estabrook和Wahlquist ${ }^{[73]}$ 计算 了引力波对探测到的脉冲星频率的影响, 随 后Detweiler ${ }^{[74]}$ 对此进行了推广, 给出了脉冲星信 号在引力波影响下到达观测者的时间变化 $R(t)$ 的 表达式. Hellings和Downs ${ }^{[75]}$ 提出通过测量脉冲星 信号到达时间残差的关联函数来测量引力波及 其偏振态, 这个关联函数随脉冲星之间夹角的曲 线也称为Hellings-Downs曲线. $\hat{P}_{+}, \hat{P}_{\times}$及 $\hat{P}_{b}$ 类光引力 波偏振态的Hellings-Downs曲线见图4. 对于更一 般的修改引力中的Hellings-Downs曲线, 可参考文 献[16, 44, 76 82]. Chamberlin和Siemens ${ }^{[79]}$ 指出, 当 两颗脉冲星夹角很小时, PTA对纵向偏振模式 $\hat{P}_{x}$, $\hat{P}_{y}$ 与 $\hat{P}_{l}$ 的灵敏度将大大高于横向偏振模式 $\hat{P}_{+}$, $\hat{P}_{\times}$与 $\hat{P}_{b}$. 脉冲星信号红移可表示为

$$
z(t, \hat{w}) \equiv \frac{v_{e}-v_{p}}{v_{p}}=\frac{\hat{p}^{i} \hat{p}^{j}}{2(1+\hat{w} \cdot \hat{p})} \Delta h_{i j},
$$

其中 $\hat{w}$ 为引力波方向, $\hat{p}$ 为地球指向脉冲星的单位矢 量, $\Delta h_{i j}=h_{i j}\left(t_{p}, \hat{w}\right)-h_{i j}\left(t_{e}, \hat{w}\right)$ 表示引力波信号在脉冲 星信号发射时刻和被接收时刻的差值. 类似地, 可以 定义脉冲星计时的模式函数

$$
F^{A}(\hat{w})=\epsilon_{i j}^{A}(\hat{w}) \frac{\hat{p}^{i} \hat{p}^{j}}{2(1+\hat{w} \cdot \hat{p})} .
$$

最近Arzoumanian等人 ${ }^{[83]}$ 对NANOGrav(北美纳 赫兹引力波天文台)长达11年的PTA数据进行分析, 给出了Hellings-Downs曲线的观测结果, 只是目前误 差太大, 还无法用来区分不同的引力波偏振态.

\section{5 结论}

引力波不影响单个检验粒子的测地线运动. 要探 


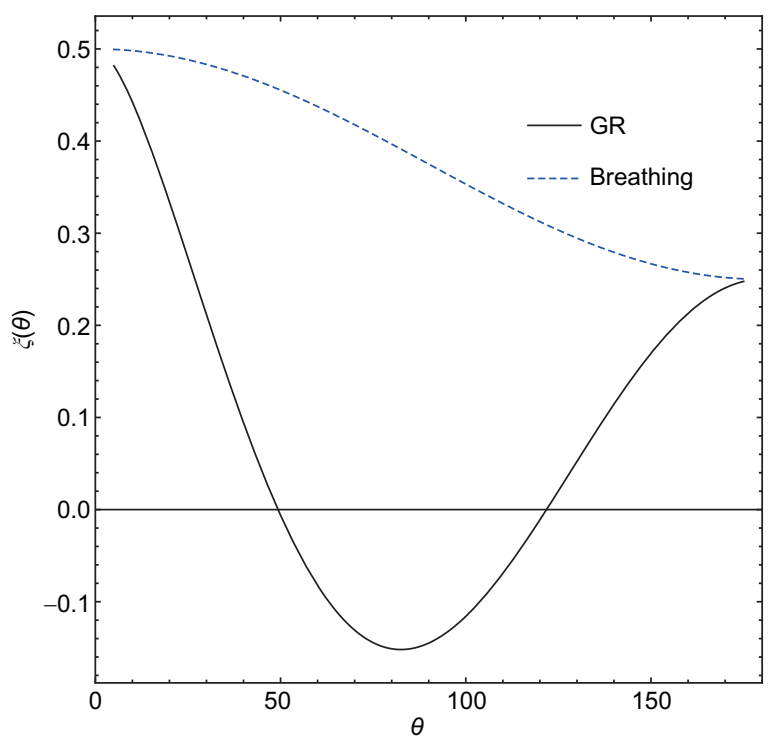

图 4 (网络版彩色) $\hat{P}_{+}, \hat{P}_{\times}$及 $\hat{P}_{\mathrm{b}}$ 类光引力波偏振态的Hellings-Downs 曲线. GR代表 $\hat{P}_{+}$与 $\hat{P}_{\times}$, Breathing 代表 $\hat{P}_{\mathrm{b}}$

Figure 4 (Color online) The Hellings-Downs curves for null gravitational waves with $\hat{P}_{+}, \hat{P}_{\times}$or $\hat{P}_{\mathrm{b}}$ polarizations. GR represents the tensor modes $\hat{P}_{+}$and $\hat{P}_{\times} ;$Breathing represents the breathing mode $\hat{P}_{\mathrm{b}}$

测引力波, 需要测量两个走测地线的相邻检验粒子 的相对运动, 即要测量引力波引起的潮汐力. 也就是 说, 需要通过测地线偏离方程来探测引力波. 本文对 引力波偏振态的定义与探测进行了简单介绍. 由于 只有黎曼张量的电分量 $R_{i 0 j 0}$ 出现在测地线偏离方程 中, 所以利用 $R_{i 0 j 0}$ 及测地线偏离方程定义引力波偏 振态. 在最一般的度规引力理论中, 可能出现 $\hat{P}_{+}, \hat{P}_{\times}$, $\hat{P}_{b}, \hat{P}_{x}, \hat{P}_{y}$ 及 $\hat{P}_{l}$ 这6种基本偏振态. 对于类光引力波,
这6种偏振模式为 6 个相互独立的偏振态, “+”偏振模 式 $\hat{P}_{+}$与“×”偏振模式 $\hat{P}_{\times}$代表自旋为 2 的张量引力波, 矢量- $x$ 模式 $\hat{P}_{x}$ 与矢量 $-y$ 模式 $\hat{P}_{y}$ 代表自旋为 1 的矢量引 力波, 而呼吸模式 $\hat{P}_{\mathrm{b}}$ 及纵模模式 $\hat{P}_{1}$ 代表标量引力波.

本文分别讨论了广义相对论、Brans-Dicke理 论、 $f(R)$ 理论、Horndeski理论、爱因斯坦-以太理论 和 $\mathrm{TeVe}$ 理论中的引力波偏振态问题. 在广义相对论 中, 只有“+”和“×”两种偏振模式, 而Brans-Dicke理论 中的无质量标量场将激发额外的呼吸偏振模式 $\hat{P}_{b}$. 与Brans-Dicke理论不同, 与 $f(R)$ 引力等效的标量张 量理论以及Horndeski理论中的有质量标量场, 会激 发一个由呼吸模与纵模混合而成的标量偏振态. 在 爱因斯坦-以太理论中, 由于矢量场的引入, 不仅产 生了两个矢量偏振模式 $\hat{P}_{x}$ 与 $\hat{P}_{y}$, 而且还产生了一个 由呼吸模与纵模混合而成的标量偏振态. 在 TeVeS 理 论中, 则有两个张量偏振模式、两个矢量偏振模式 以及两个呼吸态与纵模态的混合单态. 尽管截至目 前, 无论是激光干涉仪还是脉冲星计时阵列, 均未探 测到偏振模式的相关信息, 但未来精度更高的探测 器将会提供这方面的信息.

总之, 引力波偏振态的测量可以用来验证广 义相对论及甄别不同的引力理论. 如果只探测到 了“+”和“ ×”两种引力波偏振态, 则该结果强有力地 支持了爱因斯坦广义相对论. 如果除了上述两种偏 振态外, 也探测到了呼吸模式, 则还有无质量的标量 场在传递引力相互作用. 所以引力波偏振态的测量 可以用来探究引力的本质.

\section{参考文献}

1 Abbott B P, Abbott R, Abbott T D, et al. Observation of gravitational waves from a binary black hole merger. Phys Rev Lett, 2016, 116: 061102

2 Abbott B P, Abbott R, Abbott T D, et al. GW151226: Observation of gravitational waves from a 22-solar-mass binary black hole coalescence. Phys Rev Lett, 2016, 116: 241103

3 Abbott B P, Abbott R, Abbott T D, et al. GW170104: Observation of a 50-Solar-mass binary black hole coalescence at redshift 0.2. Phys Rev Lett, 2017, 118: 221101

4 Abbott B P, Abbott R, Abbott T D, et al. GW170608: Observation of a 19-solar-mass binary black hole coalescence. Astrophys J, 2017, 851: L35

5 Abbott B P, Abbott R, Abbott T D, et al. GW170814: A three-detector observation of gravitational waves from a binary black hole coalescence. Phys Rev Lett, 2017, 119: 141101

6 Abbott B P, Abbott R, Abbott T D, et al. GW170817: Observation of gravitational waves from a binary neutron star inspiral. Phys Rev Lett, 2017, 119: 161101

7 Hulse R A, Taylor J H. Discovery of a pulsar in a binary system. Astrophys J, 1975, 195: L51-L53

8 Gertsenshtein M E, Pustovoit V I. On the detection of low frequency gravitational waves. Sov Phys JETP, 1962, 16: 433

9 Goldstein A, Veres P, Burns E, et al. An ordinary short gamma-ray burst with extraordinary implications: Fermi-GBM detection of GRB 170817A. 
Astrophys J Lett, 2017, 848: L14

10 Savchenko V, Ferrigno C, Kuulkers E, et al. INTEGRAL detection of the first prompt gamma-ray signal coincident with the gravitational-wave event GW170817. Astrophys J Lett, 2017, 848: L15

11 Abbott B P, Abbott R, Abbott T D, et al. Gravitational waves and gamma-rays from a binary neutron star merger: GW170817 and GRB 170817A. Astrophys J, 2017, 848: L13

12 Flanagan E E, Hughes S A. The Basics of gravitational wave theory. New J Phys, 2005, 7: 204

13 Newman E, Penrose R. An approach to gravitational radiation by a method of spin coefficients. J Math Phys, 1962, 3: 566-578

14 Eardley D M, Lee D L, Lightman A P. Gravitational-wave observations as a tool for testing relativistic gravity. Phys Rev D, 1973, 8: 3308-3321

15 Liang D, Gong Y, Hou S, et al. Polarizations of gravitational waves in $f(R)$ gravity. Phys Rev D, 2017, 95: 104034

16 Hou S, Gong Y, Liu Y. The polarizations of gravitational waves in Scalar-Tensor theory. arXiv: 1704.01899

17 Brans C, Dicke R. Mach's principle and a relativistic theory of gravitation. Phys Rev, 1961, 124: 925-935

18 Nordtvedt K Jr. Post Newtonian metric for a general class of scalar tensor gravitational theories and observational consequences. Astrophys J, 1970, 161: 1059-1067

19 Wagoner R V. Scalar tensor theory and gravitational waves. Phys Rev D, 1970, 1: 3209-3216

20 Fujii Y, Maeda K. The Scalar-tensor Theory of Gravitation. Cambridge: Cambridge University Press, 2007

21 Faraoni V. Cosmology in Scalar Tensor Gravity. Berlin: Springer, 2004

22 De Felice A, Tsujikawa S. $f(R)$ theories. Living Rev Rel, 2010, 13: 3

23 Sotiriou T P, Faraoni V. $f(R)$ Theories of gravity. Rev Mod Phys, 2010, 82: 451-497

24 Buchdahl H A. Non-linear Lagrangians and cosmological theory. Mon Not Roy Astron Soc, 1970, 150: 1-8

25 O'Hanlon J. Intermediate-range gravity—A generally covariant model. Phys Rev Lett, 1972, 29: 137-138

26 Teyssandier P, Tourrenc P. The Cauchy problem for the $R+R^{2}$ theories of gravity without torsion. J Math Phys, 1983, 24: 2793

27 Horndeski G W. Second-order scalar-tensor field equations in a four-dimensional space. Int J Theor Phys, 1974, 10: 363-384

28 Ostrogradsky M. Memories on differential equations, relating to the problem of isoperimeters. Mem Acad St Petersbourg, 1850, 6: 385-517

29 Mattingly D. Modern tests of Lorentz invariance. Living Rev Rel, 2005, 8: 5

30 Jacobson T, Mattingly D. Gravity with a dynamical preferred frame. Phys Rev D, 2001, 64: 024028

31 Will C M, Nordtvedt K Jr. Conservation laws and preferred frames in relativistic gravity. I. Preferred-frame theories and an extended PPN formalism. Astrophys J, 1972, 177: 757

32 Nordtvedt K J, Will C M. Conservation laws and preferred frames in relativistic gravity. II. Experimental evidence to rule out preferred-frame theories of gravity. Astrophys J, 1972, 177: 775-792

33 Hellings R W, Nordtvedt K. Vector-metric theory of gravity. Phys Rev D, 1973, 7: 3593-3602

34 Eling C, Jacobson T. Static post Newtonian equivalence of GR and gravity with a dynamical preferred frame. Phys Rev D, 2004, 69: 064005

35 Elliott J W, Moore G D, Stoica H. Constraining the new Aether: Gravitational cerenkov radiation. J High Energy Phys, 2005, 8: 66

36 Carroll S M, Lim E A. Lorentz-violating vector fields slow the universe down. Phys Rev D, 2004, 70: 123525

37 Lim E A. Can we see Lorentz-violating vector fields in the CMB? Phys Rev D, 2005, 71: 063504

38 Armendariz-Picon C. Could dark energy be vector-like? J Cosmol Astrop Phys, 2004, 0407: 007

39 Wei H, Cai R G. Interacting vector-like dark energy, the first and second cosmological coincidence problems. Phys Rev D, 2006, 73: 083002

40 Zlosnik T G, Ferreira P G, Starkman G D. The Vector-tensor nature of Bekenstein's relativistic theory of modified gravity. Phys Rev D, 2006, 74: 044037

41 Zlosnik T G, Ferreira P G, Starkman G D. Modifying gravity with the Aether: An alternative to dark matter. Phys Rev D, 2007, 75: 044017

42 Zlosnik T G, Ferreira P G, Starkman G D. Growth of structure in theories with a dynamical preferred frame. Phys Rev D, 2008, 77: 084010

43 Jacobson T, Mattingly D. Einstein-Aether waves. Phys Rev D, 2004, 70: 024003

44 Gong Y, Hou S, Liang D, et al. Gravitational waves in Einstein-Aether theory and generalized TeVeS theory after GW170817. arXiv: 1801.03382

45 Bekenstein J D. Relativistic gravitation theory for the MOND paradigm. Phys Rev D, 2004, 70: 083509

46 Milgrom M. A modification of the Newtonian dynamics as a possible alternative to the hidden mass hypothesis. Astrophys J, 1983, 270: 365-370

47 Milgrom M. A modification of the Newtonian dynamics: Implications for galaxies. Astrophys J, 1983, 270: 371-383

48 Milgrom M. A modification of the Newtonian dynamics: Implications for galaxy systems. Astrophys J, 1983, 270: 384-389

49 Sagi E. Propagation of gravitational waves in generalized TeVeS. Phys Rev D, 2010, 81: 064031

50 Sagi E, Bekenstein J D. Black holes in the TeVeS theory of gravity and their thermodynamics. Phys Rev D, 2008, 77: 024010

51 Skordis C, Mota D F, Ferreira P G, et al. Large scale structure in Bekenstein's theory of relativistic modified newtonian dynamics. Phys Rev Lett, 2006, 96: 011301

52 Skordis C. Teves cosmology: Covariant formalism for the background evolution and linear perturbation theory. Phys Rev D, 2006, 74: 103513

53 Skordis C. Generalizing tensor-vector-scalar cosmology. Phys Rev D, 2008, 77: 123502 
54 Kramer M, Champion D J. The European pulsar timing array and the large European array for pulsars. Class Quant Grav, 2013, 30: 224009

55 Hobbs G, Archibald A, Arzoumanian Z, et al. The international pulsar timing array project: Using pulsars as a gravitational wave detector. Class Quant Grav, 2010, 27: 084013

56 McLaughlin M A. The North American nanohertz observatory for gravitational waves. Class Quant Grav, 2013, 30: 224008

57 Hobbs G. The parkes pulsar timing array. Class Quant Grav, 2013, 30: 224007

58 Desvignes G, Caballero R N, Lentati L, et al. High-precision timing of 42 millisecond pulsars with the european pulsar timing array. Mon Not Roy Astron Soc, 2016, 458: 3341-3380

59 Verbiest J P W, Lentati L, Hobbs G, et al. The international pulsar timing array: First data release. Mon Not Roy Astron Soc, 2016, 458: 1267-1288

60 Amaro-Seoane P, Audley H, Babak S, et al. Laser interferometer space antenna. arXiv: 1702.00786

61 Kawamura S, Ando M, Seto N, et al. The Japanese space gravitational wave antenna: DECIGO. Class Quant Grav, 2011, 28: 094011

62 Graham P W, Hogan J M, Kasevich M A, et al. Mid-band gravitational wave detection with precision atomic sensors. arXiv: 1711.02225

63 Luo J, Chen L, Duan H, et al. TianQin: A space-borne gravitational wave detector. Class Quant Grav, 2016, 33: 035010

64 Will C M. The confrontation between general relativity and experiment. Living Rev Rel, 2014, 17: 4

65 Chatziioannou K, Yunes N, Cornish N. Model-independent test of general relativity: An extended post-Einsteinian framework with complete polarization content. Phys Rev D, 2012, 86: 022004

66 Isi M, Weinstein A J, Mead C, et al. Detecting beyond-Einstein polarizations of continuous gravitational waves. Phys Rev D, 2015, 91 : 082002

67 Isi M, Pitkin M, Weinstein A J. Probing dynamical gravity with the polarization of continuous gravitational waves. Phys Rev D, 2017, $96: 042001$

68 Nishizawa A, Taruya A, Hayama K, et al. Probing non-tensorial polarizations of stochastic gravitational-wave backgrounds with ground-based laser interferometers. Phys Rev D, 2009, 79: 082002

69 Callister T, Biscoveanu A S, Christensen N, et al. Polarization-based tests of gravity with the stochastic gravitational-wave background. Phys Rev X, 2017, 7: 041058

70 Weber J. Gravitational radiation from the pulsars. Phys Rev Lett, 1968, 21: 395-396

71 Ostriker J P, Gunn J E. On the nature of pulsars. 1. Theory. Astrophys J, 1969, 157: 1395-1417

72 Abbott B P, Abbott R, Abbott T D, et al. First search for nontensorial gravitational waves from known pulsars. Phys Rev Lett, 2018, 120 : 031104

73 Estabrook F B, Wahlquist H D. Response of doppler spacecraft tracking to gravitational radiation. Gen Rel Grav, 1975, 6: 439-447

74 Detweiler S L. Pulsar timing measurements and the search for gravitational waves. Astrophys J, 1979, 234: 1100-1104

75 Hellings R W, Downs G S. Upper limits on the isotropic graviational radiation background from pulsar timing analysis. Astrophys J, 1983, 265: L39-L42

76 Lee K J, Jenet F A, Price R H. Pulsar timing as a probe of non-einsteinian polarizations of gravitational waves. Astrophys J, 2008, 685: 1304-1319

77 Lee K, Jenet F A, Price R H, et al. Detecting massive gravitons using pulsar timing arrays. Astrophys J, 2010, 722: 1589-1597

78 Lee K J. Pulsar timing arrays and gravity tests in the radiative regime. Class Quant Grav, 2013, 30: 224016

79 Chamberlin S J, Siemens X. Stochastic backgrounds in alternative theories of gravity: Overlap reduction functions for pulsar timing arrays. Phys Rev D, 2012, 85: 082001

80 Yunes N, Siemens X. Gravitational-wave tests of general relativity with ground-based detectors and pulsar timing-arrays. Living Rev Rel, 2013, 16: 9

81 Gair J, Romano J D, Taylor S, et al. Mapping gravitational-wave backgrounds using methods from CMB analysis: Application to pulsar timing arrays. Phys Rev D, 2014, 90: 082001

82 Gair J R, Romano J D, Taylor S R. Mapping gravitational-wave backgrounds of arbitrary polarisation using pulsar timing arrays. Phys Rev D, 2015, 92: 102003

83 Arzoumanian Z, Bakeer P T, Brazier A, et al. The NANOGrav 11-year data set: Pulsar-timing constraints on the stochastic gravitational-wave background. arXiv: 1801.02617 


\title{
The polarizations of gravitational waves
}

\author{
Qing $\mathrm{Gao}^{1}$, Yungui Gong ${ }^{2 *} \&$ Dicong Liang ${ }^{2}$ \\ ${ }^{1}$ School of Physical Science and Technology, Southwest University, Chongqing 400715, China; \\ ${ }^{2}$ School of Physics, Huazhong University of Science and Technology, Wuhan 430074, China \\ *Corresponding author, E-mail: yggong @ hust.edu.cn
}

The direct detection of gravitational waves opens a new window to probe the nature of gravity, and it brings us into the era of multi-messenger astronomy. Gravitational wave has no effect on the geodesics of a test particle, but it affects the relative motion between two nearby test particles. To measure gravitational wave and its polarizations, we need to study the geodesic deviation equation for two nearby test particles. Since only the electric part of the Riemann tensor $R_{i 0 j 0}$ appears in the geodesic deviation equation and it has six independent components, there are up to six possible polarization states in general metric theories of gravity. In terms of the electric part of the Riemann tensor, the six polarizations are defined as follows. $\hat{P}_{+}=-R_{x 0 x 0}+R_{y 0 y 0}$ corresponds to the "+" mode, $\hat{P}_{\times}=2 R_{x 0 y 0}$ denotes the "X" mode, $\hat{P}_{b}=R_{x 0 x 0}+R_{y 0 y 0}$ corresponds to the breathing mode, $\hat{P}_{x}=R_{x 0 z 0}$ corresponds to the vector- $x$ mode, $\hat{P}_{x}=R_{y 0 z 0}$ represents to the vector- $y$ mode, and $\hat{P}_{l}=R_{z 0 z 0}$ denotes the longitudinal mode. For weak, plane and null gravitational waves, these six polarizations can be classified based on their properties under the Lorentz transformation. In Einstein's general relativity, the gravitational waves are tensor waves and there are "+" and " $\times$ " modes of polarization. In Brans-Dicke theory, in addition to the "+" and " $\times$ " polarization states, the scalar breathing mode also appears, and all these three polarizations are transverse modes. In the most general scalar-tensor theory of gravity, the Horndeski theory, the massive scalar field excites both the breathing and the longitudinal modes, so in addition to the "+" and " $\times$ " polarizations, there exists a single mix state consisting of both the breathing and the longitudinal modes. In the massless limit, the longitudinal mode disappears and the mix state becomes the pure transverse breathing polarization as that in Brans-Dicke theory. The nonlinear $f(R)$ theory of gravity can be written as a scalar-tensor theory of gravity, so the polarization states in $f(R)$ gravity are the same as those in Horndeski theory except that there is no massless limit in $f(R)$ gravity. In Einstein-Aether theory, due to the existence of the unit timelike vector field, the local Lorentz invariance is violated. The gravitational waves propagate with speeds different from the speed of light, and there are five independent polarization states, the "+" state $\hat{P}_{+}$, the " $\times$" state $\hat{P}_{\times}$, the vector $-x$ state $\hat{P}_{x}$, the vector $-y$ state $\hat{P}_{y}$ and the mix state consisting of both the breathing and the longitudinal modes excited by the scalar field. In TeVeS theory, the local Lorentz invariance is also broken, so the gravitational waves in TeVeS theory propagate with speeds different from the speed of light. There are six polarization states in TeVeS theory, $\hat{P}_{+}, \hat{P}_{\times}, \hat{P}_{x}, \hat{P}_{y}$ and two mix states excited by the two scalar fields. Each of the mix states consists of both the breathing and the longitudinal modes. Because gravitational waves have different polarization states for different theories of gravity, the measurement of polarizations is a powerful tool in probing the nature of gravity.

polarizations of gravitational waves, scalar-tensor theory of gravity, Einstein-Aether theory, tensor-vector-scalar theory

doi: 10.1360/N972018-00167 